\title{
A service development study of the assessment and management of fracture risk in Parkinson's disease
}

\author{
Samuel Shribman · Kelli M. Torsney • \\ Alastair J. Noyce • Gavin Giovannoni • \\ Julian Fearnley $\cdot$ Ruth Dobson
}

Received: 14 January 2014/Revised: 25 March 2014/ Accepted: 26 March 2014/Published online: 10 April 2014

(c) Springer-Verlag Berlin Heidelberg 2014

\begin{abstract}
Parkinson's disease (PD) is associated with an increased risk of fragility fracture. FRAX and Qfracture are risk calculators that estimate the 10-year risk of hip and major fractures and guide definitive investigation for osteoporosis using dual X-ray absorptiometry (DEXA) imaging. It is unclear which PD patients should be considered for fracture risk assessment and whether FRAX or Qfracture should be used. Seventy-seven patients with PD were recruited in the movement disorders clinic. Data were collected on PD-related characteristics and fracture risk scores were calculated. Patients with previous osteoporotic fractures had a higher incidence of falls $(p=0.0026)$ and use of bilateral walking aids $(p=0.0187)$ in addition to longer disease duration $(p=0.0037)$. Selecting patients with falls in combination with either disease duration $>5$ years, bilateral walking aids, or previous osteoporotic fracture distinguished patients with and without previous osteoporotic fracture with specificity $67.7 \%(95 \%$ CI 55.0-78.8) and sensitivity $100.0 \%$ (95\% CI 73.5-100.0). Qfracture calculated significantly higher fracture risk
\end{abstract}

S. Shribman $(\bowtie) \cdot$ A. J. Noyce · G. Giovannoni · R. Dobson Blizard Institute, Barts and the London School of Medicine and Dentistry, Queen Mary University London, 4 Newark Street, London E1 2AT, UK

e-mail: samshribman@gmail.com

S. Shribman · A. J. Noyce · G. Giovannoni · J. Fearnley Royal London Hospital, Barts Health NHS Trust, Whitechapel, London, UK

K. M. Torsney

West Middlesex University Hospital NHS Trust, London, UK

A. J. Noyce

Reta Lila Weston Institute of Neurological Studies,

UCL Institute of Neurology, London, UK scores than FRAX for hip $(p<0.0001)$ and major $(p=0.0008)$ fracture in PD patients. Receiver operating characteristic curves demonstrated that FRAX outperformed Qfracture with an area under the curve of 0.84 (95\% CI $0.70-0.97, p=0.0004)$ for FRAX and 0.68 (95\% CI 52-86, $p=0.0476$ ) for Qfracture major fracture risk calculators. We suggest that falls in combination with either a disease duration longer than 5 years or bilateral walking aids or previous osteoporotic fracture should be used as red flags in PD patients to prompt clinicians to perform a FRAX fracture risk assessment in the neurology clinic.

Keywords Parkinson's disease · Fracture · Risk calculation · Osteoporosis

\section{Introduction}

Parkinson's disease (PD) is associated with an increased risk of fragility fracture $[1,2]$. The Global Longitudinal Study of Osteoporosis in Women (GLOW) found the association of fracture with PD was stronger than for any other co-morbidity [3]. The 2-year fracture rate was $16 \%$ in female PD patients, with an age-adjusted hazard ratio for incident fracture of 2.2 in PD patients compared to controls. This has been accounted for by a higher incidence of both falls and reduced bone mineral density in PD [4].

Falls increase in frequency with disease progression, and meta-analyses suggest that $46 \%$ of all PD patients fall within a 3-month period [5]. Several motor and non-motor features have been identified as contributing to falls, including postural instability, gait disturbance, cognitive impairment, postural hypotension and urinary symptoms $[6,7]$. Additionally, reduced bone mineral density is 
common in PD and can be diagnosed using dual X-ray absorptiometry (DEXA) imaging [8]. A recent study of 186 patients with early PD demonstrated that $11.8 \%$ of patients had osteoporosis (T-score less than -2.5 ) and $41.4 \%$ had osteopenia (T-score between -1 and -2.5 ) [9]. Immobility, vitamin D deficiency, use of dopaminergic treatments and reduced nutritional intake contribute to reduced bone mineral density in PD.

Fragility fractures are a significant cause of morbidity. Measures to assess and reduce fracture risk in PD are essential [10]. Several fracture risk calculators, including FRAX and Qfracture, have been validated and are freely available via the internet. These estimate the 10-year risk of either hip fracture or major fracture using clinical risk factors for osteoporosis, including the body mass index (Table 1). FRAX was developed using nine international population-based prospective cohorts [11], whereas Qfracture was derived from prospective primary care data collected in the UK [12]. In contrast to FRAX, the

Table 1 Data used in the calculation of FRAX and Qfracture risk scores

\begin{tabular}{ll}
\hline FRAX & Qfracture \\
\hline Age & Age \\
Sex & Sex \\
Weight, height; BMI & Weight, height; BMI \\
Previous fracture & Previous fragility fracture \\
Parental hip fracture & Parental osteoporosis or hip fracture \\
Current smoking & Current/previous smoking, number of \\
& cigarettes \\
Glucocorticoid exposure & Regular glucocorticoid exposure \\
Rheumatoid arthritis & Rheumatoid arthritis or SLE \\
Secondary osteoporosis & Alcohol units/day \\
Alcohol (>3 units/day) & Ethnicity \\
Femoral DEXA & Diabetes \\
(if available) & Nursing/care home residence \\
& Falls \\
& Dementia \\
& Cancer \\
& Asthma/COPD \\
& Heart attack, angina, stroke or TIA \\
& Chronic liver disease \\
& Chronic kidney disease \\
& Parkinson's disease \\
& Malabsorption including Crohn's disease \\
& Endocrine problems \\
& Epilepsy/anticonvulsant exposure \\
& Anti-depressants \\
& Oestrogen-only HRT \\
\hline & \\
& \\
& \\
& \\
&
\end{tabular}

Qfracture algorithm includes PD as a specific risk factor for osteoporotic fractures.

The National Institute for Clinical Excellence (NICE) recommends that fracture risk assessment using either FRAX or Qfracture should be considered in all patients with possible secondary osteoporosis, and that these should be used to determine who should undergo formal bone mineral density measurement using DEXA imaging [13]. Recommendations on the risk threshold at which a patient requires this investigation are not included. Guidelines on the primary prevention of osteoporotic fractures state that those patients with osteoporosis ( $\mathrm{T}$-score less than -2.5 ) should be offered bisphosphonates as first-line treatment if they have an appropriate number of clinical risk factors for osteoporosis according to their age group [14].

There remains uncertainty as to which PD patients should have an assessment of their fracture risk. Moreover, FRAX or Qfracture have not been directly compared in PD patients. This service development study firstly aims to establish red flags, specific to PD patients, which should prompt a fracture risk assessment using a risk calculator. Secondly, it aims to determine which risk calculator should be used in this patient group in the outpatient clinic.

\section{Methods}

This study was approved by the Clinical Effectiveness Unit at Barts Health NHS Trust (project reference 578-13). PD patients diagnosed according to the Queen Square Brain Bank criteria [15] were assessed. Sufficient data for FRAX and Qfracture risk calculations were collected (Table 1) through patient interviews and a review of the medical records following informed consent from each patient. Data were also collected on previous osteoporotic fractures, falls, duration of disease, disease severity (using Hoehn-Yahr stage), motor fluctuations, gait freezing, urinary symptoms, cognitive impairment, use of dopaminergic drugs and osteoporosis-related outcome measures including previous DEXA imaging and treatment with bisphosphonates.

Fracture risk scores were calculated using FRAX and Qfracture 10-year risk for both "hip" and "major" (hip, wrist, shoulder or vertebra) fracture. Osteoporotic fracture is defined by FRAX as any fracture either occurring spontaneously in adult life, or arising from trauma, which in a healthy individual would not have resulted in fracture. However, Qfracture defines osteoporotic fracture as a "major fracture" occurring in this context. Satisfaction of both of these criteria was required in order to classify a patient as having a previous osteoporotic fracture for this study. In those patients without a recorded body mass index and unable to give their height and weight, these were 
measured and body mass index was calculated according to a standard formula. There is an upper weight limit for FRAX and those patients exceeding this weight were recorded at the maximum $125 \mathrm{~kg}$.

Categorical data on PD-specific characteristics of those with or without previous osteoporotic fracture were compared using Fisher's exact test. Data distributions were assessed for normality using the Shapiro-Wilk test. Nonparametric unpaired group data were compared using the Mann-Whitney $U$ test and paired data with the Wilcoxon signed-rank matched pairs test. Agreement between individual scores and systematic error between risk calculators was assessed using Bland-Altman plots. Receiver operating characteristic (ROC) curves were plotted to compare the trade-off between sensitivity and specificity for each fracture risk calculator in distinguishing patients with or without previous osteoporotic fractures. The area under the curve (AUC) was calculated as measure of the performance of each risk calculator. The sensitivities and cut-off values for each fracture risk calculator in distinguishing patients with and without previous osteoporotic fracture were also compared at specificities of 80,85 and $90 \%$. Statistical analysis was performed using GraphPad Prism Version 6.

\section{Results}

Data were collected on 78 patients, one of whom was excluded on the basis that the diagnosis was changed to multi-system atrophy at a subsequent clinic visit. The mean age was 66.7 years (SD 10.3, range 43-85) and $64.9 \%$ of the subjects were male, consistent with the gender distribution of PD patients in the UK [16]. The median disease duration was 6 years (range 0-30) and the median Hoehn-
Yahr stage was 2 (range 1-5). Levodopa treatment was recorded for $97.4 \%$ and dopamine agonists for $48.6 \%$ of patients at the time of data collection. History of osteoporotic fracture was recorded for $15.6 \%$ of patients, with $83.3 \%$ of these occurring after PD diagnosis. Of these 12 patients, three had undergone DEXA imaging in primary care and were taking bisphosphonates. Multiple osteoporotic fractures since diagnosis were recorded in the same three patients (Table 2).

In comparing those patients with and without a previous osteoporotic fracture, there were several PD-related characteristics that differed significantly between the two groups. Falls occurred in all patients with a previous osteoporotic fracture, compared to $55 \%$ of patients without a previous fracture $(p=0.0026)$. In all patients with previous osteoporotic fractures at least one fracture had been associated with falls. Those patients with previous osteoporotic fractures had longer disease duration; median disease duration was 8.5 years in those with osteoporotic fracture compared to 5.0 years in those without $(p=0.0037)$. The use of walking aids was not significantly different between the two groups $(p=0.11)$. However, $50 \%$ of those in the fracture group used bilateral walking aids, defined as two sticks, a frame or a wheelchair, compared to $16 \%$ of patients without a previous osteoporotic fracture $(p=0.019)$. The Hoehn-Yahr stage, the use of levodopa and the presence of motor fluctuations, gait freezing, cognitive impairment or urinary frequency were not significantly different between the two groups (Table 3).

Selecting patients with falls in combination with both disease duration longer than 5 years and use of bilateral walking aids distinguished those with and without previous osteoporotic fracture with specificity of $95.2 \%$ (95\% CI
Table 2 Osteoporotic fractures recorded in this cohort

\begin{tabular}{|c|c|c|c|c|c|c|c|c|}
\hline \multirow{3}{*}{$\begin{array}{l}\text { Age (years) and } \\
\text { gender }\end{array}$} & \multirow{3}{*}{$\begin{array}{l}\text { Site of osteoporotic } \\
\text { fractures }\end{array}$} & \multicolumn{4}{|c|}{10 -year fracture risk $(\%)$} & \multirow[t]{3}{*}{ Falls } & \multirow{3}{*}{$\begin{array}{l}\text { Bilateral } \\
\text { walking aid }\end{array}$} & \multirow{3}{*}{$\begin{array}{l}\text { Disease } \\
\text { duration } \\
\text { (years) }\end{array}$} \\
\hline & & \multicolumn{2}{|l|}{ FRAX } & \multicolumn{2}{|c|}{ Qfracture } & & & \\
\hline & & Major & Hip & Major & Hip & & & \\
\hline $73 \mathrm{~m}$ & Hip & 22.0 & 18.0 & 99.9 & 99.9 & $\checkmark$ & $\boldsymbol{V}$ & 10 \\
\hline $79 f$ & Hip & 25.0 & 8.8 & 37.4 & 37.4 & $\checkmark$ & $\boldsymbol{V}$ & 2 \\
\hline $73 \mathrm{~m}$ & Shoulder & 11.0 & 4.9 & 24.2 & 24.2 & $\boldsymbol{V}$ & & 10 \\
\hline $76 f$ & Shoulder & 62.0 & 54.0 & 82.6 & 76.1 & $\checkmark$ & & 8 \\
\hline $74 f$ & Shoulder, vertebra & 32.0 & 15.0 & 22.9 & 20.1 & $\checkmark$ & $\boldsymbol{V}$ & 12 \\
\hline $79 \mathrm{f}$ & Shoulder, wrist & 37.0 & 18.0 & 69.9 & 69.9 & $\boldsymbol{V}$ & & 7 \\
\hline $69 \mathrm{~m}$ & Vertebra & 10.0 & 2.7 & 7.0 & 4.8 & $\checkmark$ & $\checkmark$ & 30 \\
\hline $78 \mathrm{f}$ & Vertebra & 24.0 & 8.6 & 14.0 & 13.4 & $\checkmark$ & $\boldsymbol{V}$ & 8 \\
\hline $76 f$ & Wrist & 19.0 & 5.5 & 6.5 & 6.5 & $\checkmark$ & $\checkmark$ & 20 \\
\hline $61 \mathrm{~m}$ & Wrist & 5.2 & 0.6 & 2.5 & 1.2 & $\checkmark$ & & 8 \\
\hline $84 \mathrm{~m}$ & Wrist & 13.0 & 7.0 & 72.6 & 72.6 & $\checkmark$ & & 6 \\
\hline $65 \mathrm{~m}$ & Wrist, vertebra & 11.0 & 2.5 & 32.4 & 32.4 & $\boldsymbol{V}$ & & 9 \\
\hline
\end{tabular}


Table 3 Comparison of PD-related characteristics in those patients with and without previous osteoporotic fracture

\begin{tabular}{lcccc}
\hline & All patients $(n=77)$ & $\begin{array}{l}\text { Patients with previous } \\
\text { osteoporotic fracture }(n=12)\end{array}$ & $\begin{array}{l}\text { Patients without previous } \\
\text { osteoporotic fracture }(n=65)\end{array}$ \\
\hline Falls & $62 \%(48)$ & $100 \%(12)$ & $55 \%(36)$ & 5 \\
Disease duration (years, median) & 6 & 8.5 & $16 \%(10)$ & 0.0026 \\
Bilateral walking aid & $22 \%(16)$ & $50 \%(6)$ & 2 & 0.0037 \\
Hoehn-Yahr stage (median) & 2 & 3 & $43 \%(20)$ & 0.0187 \\
Walking aid & $44 \%(32)$ & $58 \%(7)$ & $57 \%(37)$ & 0.1120 \\
Urinary frequency & $47 \%(36)$ & $67 \%(8)$ & $18 \%(12)$ & 0.2077 \\
Motor fluctuations & $56 \%(43)$ & $50 \%(6)$ & $46 \%(30)$ & 0.3641 \\
Cognitive impairment & $19 \%(15)$ & $25 \%(3)$ & $97 \%(63)$ \\
Gait freezing & $47 \%(36)$ & $100 \%(12)$ & 0.6928 \\
Levodopa treatment & $97 \%(75)$ & 1.0000 \\
\hline
\end{tabular}

Percentages are shown with actual numbers in brackets. The Mann-Whitney test was used to determine the $p$ values for disease duration and Hoehn-Yahr stage. The Fisher's exact test was used to determine the $p$ values for the remaining characteristics

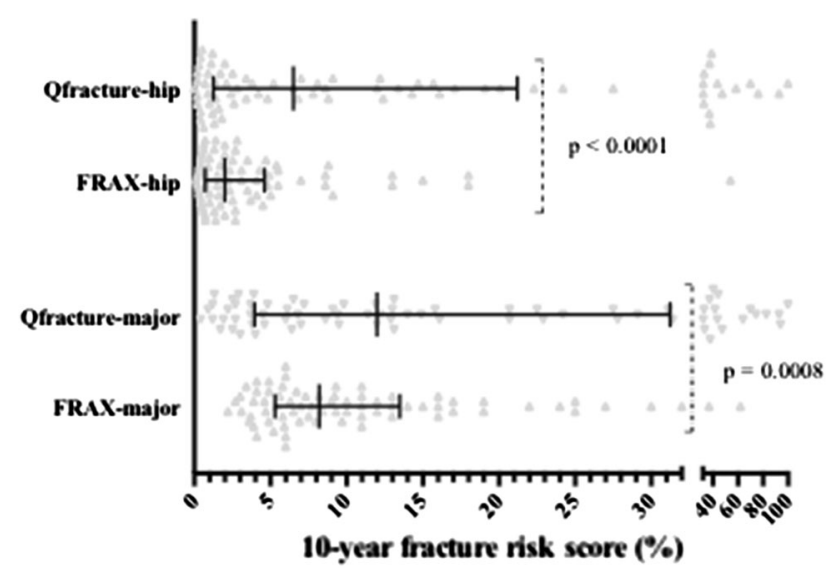

Fig. 1 Scatter plot of 10-year fracture risk scores for each calculator. The error bars represent the interquartile range and median. The Wilcoxon matched pairs signed-rank test was used to determine the $p$ values

86.7-99.0) and sensitivity of $41.7 \%$ (95\% CI 15.2-72.3). When falls in combination with either disease duration longer than 5 years or use of bilateral walking aids was used, this gave a specificity of $67.7 \%$ (95\% CI 55.0-78.8) and sensitivity of $100.0 \%$ (95\% CI 73.5-100.0). This result was unchanged when considering patients with falls in combination with either disease duration longer than 5 years or use of bilateral walking aids or a previous osteoporotic fracture.

The 10-year hip and major fracture risk calculations were compared between FRAX and Qfracture for each PD patient. The Qfracture risk scores were significantly higher than the FRAX risk scores for both hip fractures $(p<0.0001)$ and major fractures $(p=0.0008)$ (Fig. 1). The median major fracture risk score was 12.0 (range 0.4-99.9) with Qfracture and 8.2 (range 2.2-62.0) with
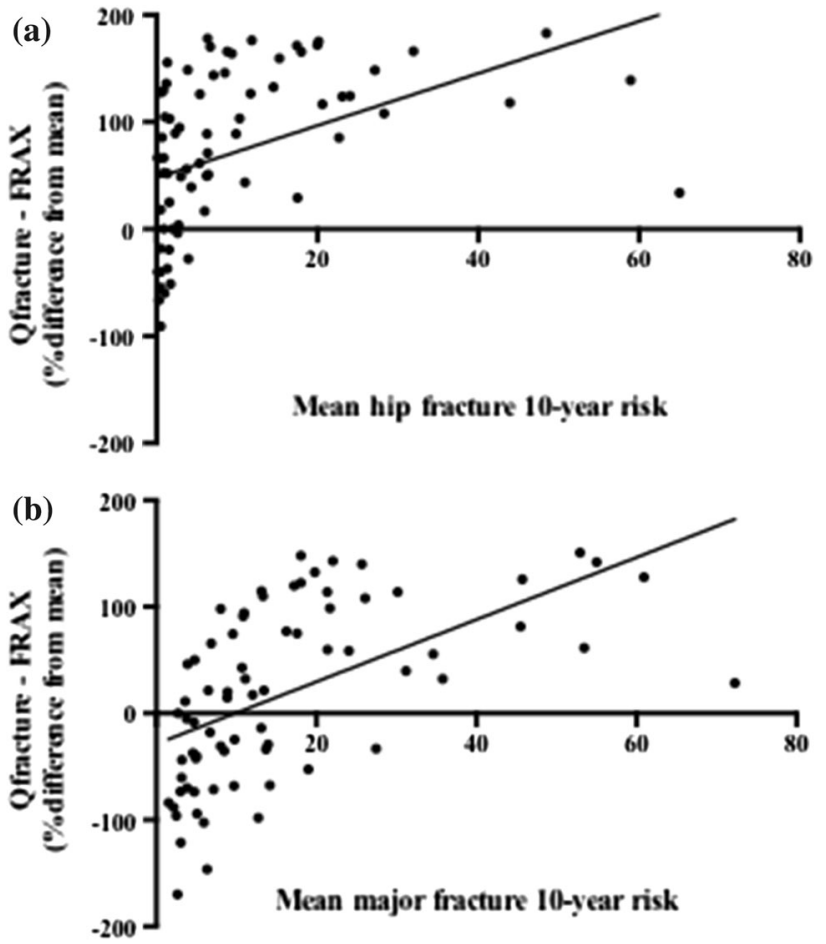

Fig. 2 Bland-Altman plots comparing FRAX and Qfracture risk scores for a hip fracture and $\mathbf{b}$ major fracture. The mean fracture risk represents that of the FRAX and Qfracture risk scores for each individual patient

FRAX. The median hip fracture risk score was 6.5 (range 0.0-99.9) with Qfracture and 2.0 (range 0.1-54.0) with FRAX. Bland-Altman plots for both hip and major fracture risk demonstrate that fracture risk calculations are relatively similar between FRAX and Qfracture in patients with low fracture risks, but that the difference between the calculations is much greater in patients at higher risks of fracture (Fig. 2). 


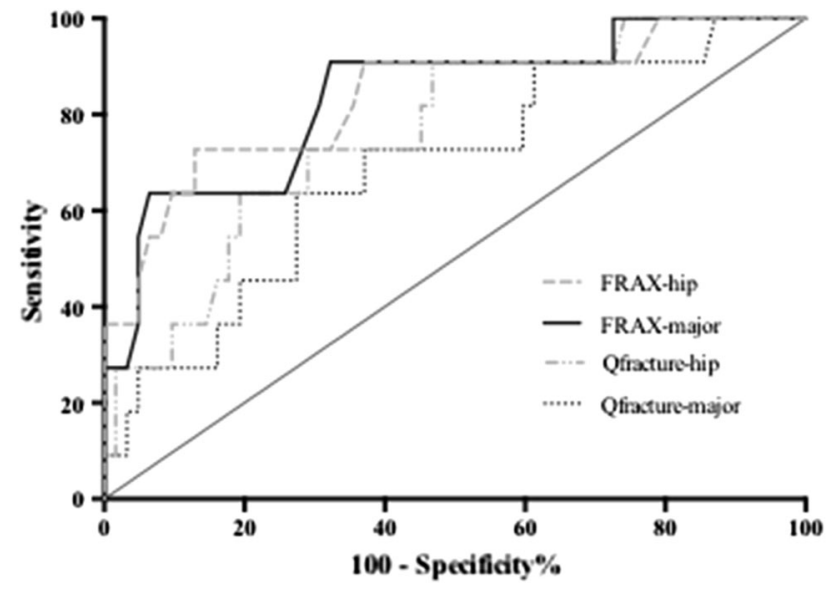

Fig. 3 Receiver operating characteristics (ROC) curves for each fracture risk calculator in distinguishing patients with or without previous osteoporotic fracture

When comparing the risk calculations from each calculator between those with or without previous osteoporotic fracture, ROC curves for each risk calculator illustrate that the FRAX calculators, for hip or major fractures, appear to be superior to the Qfracture calculator (Fig. 3). The FRAX major fracture and hip fracture risk calculators performed similarly with an AUC for FRAX major fracture of 0.84 (95\% CI $0.70-0.97, p=0.0004$ ) and an AUC for FRAX hip fracture of $0.84(95 \% \mathrm{CI}$ $0.69-0.98, \quad p=0.0004)$. AUC was $0.68 \quad(95 \%$ CI $0.52-0.86, p=0.048)$ for the Qfracture major calculator and $0.76(95 \%$ CI $0.62-0.91, p=0.0055)$ for the Qfracture hip calculator. In addition, fracture risk calculations were performed without the contribution from previous fracture, and the resulting fracture risk values were used to generate ROC curves for each calculator. Using this approach, the FRAX AUC values remained higher than those of Qfracture with a FRAX major fracture AUC of 0.73 (95\% CI $0.55-0.90, p=0.012$ ) and FRAX hip fracture AUC of 0.77 (95\% CI 0.61-0.94, $p=0.0029)$. When comparing the sensitivity of each fracture risk calculator at given specificities, FRAX had higher sensitivity than Qfracture at specificities of 80,85 and $90 \%$ (Table 4).

\section{Discussion}

Falls were present in all patients with an osteoporotic fracture. Additionally, longer disease duration and use of bilateral walking aids were significantly more common in those with fractures compared to those without. The combination of these factors performed well (sensitivity and specificity) in identifying those patients that developed fractures and can be used as "red flags" to prompt clinicians to perform fracture risk assessments. We recommend fracture risk assessment in all PD patients with falls who either use bilateral walking aids, have a disease duration $>5$ years or who have had a previous osteoporotic fracture (Fig. 4).

In contrast to a recent study by Cheng et al. [17] we did not find an association with Hoehn-Yahr stage or indeed with the presence of motor fluctuations, gait freezing, cognitive impairment or urinary frequency; these are not, therefore, included as red flags. The limited sample size and small number of patients with previous osteoporotic fractures may explain why some of these potential predictors were not significant. Importantly, using bilateral walking aids and disease duration as red flags alone identified all patients with previous osteoporotic fracture in this study, as reflected by $100 \%$ sensitivity (Table 2). Nonetheless, it is important to include previous osteoporotic fracture itself as a red flag given that it clearly increases the risk of further fractures. From our cohort, the proposed red flags would prompt fracture risk assessment in $44 \%$ of PD patients in the movement disorders clinic; however, this is likely to be less in a general neurology clinic.

An important question is whether all patients with PD should have a fracture risk assessment. Given that the use of a fracture risk calculator requires a risk threshold above which one would decide to investigate using DEXA imaging, an appreciation of the number of patients above a given threshold is required to understand the cost implications of calculating fracture risk on all PD patients. The National Osteoporosis Guideline Group (NOGG) who developed FRAX suggest that intervention with alendronate is cost-effective above a FRAX major fracture risk
Table 4 Sensitivities and cutoff values for each fracture risk calculator at specificities of 80 , 85 and $90 \%$

The $95 \%$ confidence intervals are given

\begin{tabular}{|c|c|c|c|c|c|c|}
\hline & \multicolumn{2}{|l|}{$80 \%$ specificity } & \multicolumn{2}{|l|}{$85 \%$ specificity } & \multicolumn{2}{|l|}{$90 \%$ specificity } \\
\hline & Sensitivity & Cut-off & Sensitivity & Cut-off & Sensitivity & Cut-off \\
\hline FRAX-major & $63.6(30.8-89.1)$ & $>13.5$ & $63.6(30.8-89.1)$ & $>15.5$ & $63.6(30.8-89.1)$ & $>16.5$ \\
\hline Qfracture-major & $45.5(16.8-76.2)$ & $>31.9$ & $27.3(6.0-61.0)$ & $>31.9$ & $27.3(6.0-61.0)$ & $>44.0$ \\
\hline FRAX-hip & $72.7(39.0-94.0)$ & $>4.2$ & 72.7 (39.0-94.0) & $>4.75$ & $63.6(30.8-89.1)$ & $>5.5$ \\
\hline Qfracture-hip & $63.6(30.8-89.1)$ & $>19.6$ & $36.4(10.9-69.2)$ & $>32.55$ & $36.4(10.9-69.2)$ & $>37.3$ \\
\hline
\end{tabular}




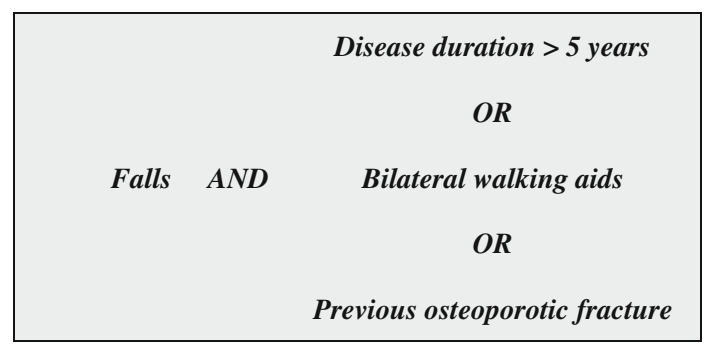

Fig. 4 Red flags to prompt fracture risk assessment in PD

threshold of $7 \%$ [18]. By way of example, a threshold with the FRAX major fracture risk calculator of $10 \%$ would amount to around one-third of all PD patients undergoing DEXA imaging in our cohort, around half of whom have red flags. At higher thresholds, multiple patients with red flags would not have qualified for imaging. In the absence of clear guidance, the implementation of the aforementioned red flag system is a cost-effective approach to identifying those PD patients that require DEXA imaging.

When comparing different fracture risk calculators, Qfracture produced significantly higher risk scores than FRAX, particularly in high-risk patients. The opposite is seen when comparing these calculators in patients with multiple sclerosis (MS) and this may be accounted for the by the inclusion of PD but not MS in Qfracture [19]. Despite this, the FRAX calculators appeared to be superior to the Qfracture calculators in this cohort, as measured by the area under the ROC curves and the comparison of the sensitivities of each calculator at given specificities. A limitation of this analysis, in addition to the size of the study, is that to assess the performance of each calculator, we compared the risk scores from those patients with previous osteoporotic fractures to those without; previous osteoporotic fracture is, in itself, an input for each of the calculators. One might, therefore, argue that different weightings for this variable between each calculator could account for the different risk scores. However, if the risk scores from these two groups are again compared, but all patients are classified as without previous fracture (for the purposes of the calculation) then FRAX continues to outperform Qfracture.

These findings are counter-intuitive given that Qfracture includes 26 input variables, including PD and falls, whereas FRAX uses only 12 variables and does not include PD or falls. One possible explanation is that our cohort, consisting of patients requiring regular specialist follow-up, may have more complex disease compared to the primary care population used to derive and validate Qfracture. Falls, dementia, anti-depressant use and nursing home residence are each included as risk factors in Qfracture and are more likely to occur in advanced PD. The use of Qfracture in patients with advanced PD could, therefore, create a tendency to overestimate fracture risk. Regardless, given the significant disparity between risk scores in PD patients using FRAX and Qfracture consistent use of one calculator is required to stratify patients in this population according to fracture risk. On the basis of the results above, we would recommend the use of the FRAX calculators in the neurology outpatient clinic. This study did not examine the use of these calculators in primary care and we would, therefore, advocate empirical use of Qfracture in PD patients in this setting until more evidence is available.

Osteoporotic fracture can be catastrophic for PD patients, and identifying those at the highest risk is of the utmost importance to their management [10]. The use of fracture risk calculators to guide decisions regarding DEXA imaging, and therefore, pharmacotherapy, is crucial; however, the value of non-pharmacological measures in reducing fracture risk in PD should not be underestimated [20]. These include exercise programmes, dietary advice, smoking cessation, medication review and measures to reduce the risk of falls such as occupational and physiotherapy, visual assessment and falls education and risk management [2]. Alternative causes of secondary osteoporosis such as hyperthyroidism, hyperparathyroidism, osteomalacia and hypogonadism should also be considered prior to initiating bisphosphonate therapy. Serum calcium and vitamin D levels should be corrected and those at risk of reduced dietary intake should receive supplementation. NICE guidance recommends alendronic acid as a first-line bisphosphonate [14], and those patients who cannot comply with instructions to remain upright for at least 30 min following administration (prior to breakfast), those with persistent upper gastrointestinal disturbance as a result of alendronate and those with contraindications such as oesophageal disease that delays emptying should receive risedronate or etidronate. In patients with $\mathrm{PD}$, care should be taken in those with dysphagia who may exhibit delayed oesophageal emptying.

Ultimately, an integrated approach including pharmacological and non-pharmacological measures to improve bone mineral density and falls reduction strategies in combination with fracture risk assessments is required to prevent osteoporotic fractures in PD [4].

Acknowledgments The authors thank Caroline Budu (Parkinson's disease nurse specialist at Bart Health NHS trust) for helping to identify study participants in the outpatient clinic.

Conflicts of interest The authors declare that they have no conflict of interest.

Ethical standards This study was approved by the Clinical Effectiveness Unit at Barts Health NHS Trust (project reference 578-13) and all subjects gave informed consent. 


\section{References}

1. Bhattacharya RK, Dubinsky RM, Lai SM, Dubinsky H (2012) Is there an increased risk of hip fracture in Parkinson's disease? A nationwide inpatient sample. Mov Disord: Off J Mov Disord Soc 27:1440-1443

2. Torsney KM, Noyce AJ, Doherty KM et al (2014) Bone health in Parkinson's disease: a systematic review and meta-analysis. J Neurol Neurosurg Psychiatry. doi:10.1136/jnnp-2013-307307

3. Dennison EM, Premaor M, Flahive J et al (2011) Effect of comorbidities on fracture risk: findings from the glow study. Osteoporos Int 22:S529-S530

4. Dobson R, Yarnall A, Noyce AJ, Giovannoni G (2013) Bone health in chronic neurological diseases: a focus on Multiple sclerosis and Parkinsonian syndromes. Pract Neurol 13:70-79

5. Pickering RM, Grimbergen YAM, Rigney U et al (2007) A metaanalysis of six prospective studies of falling in Parkinson's disease. Mov Disord 22:1892-1900

6. Wood BH, Bilclough JA, Bowron A, Walker RW (2002) Incidence and prediction of falls in Parkinson's disease: a prospective multidisciplinary study. J Neurol Neurosurg Psychiatry 72:721-725

7. Kerr GK, Worringham CJ, Cole MH et al (2010) Predictors of future falls in Parkinson disease. Neurology 75:116-124

8. Zhao Y, Shen L, Ji H-F (2013) Osteoporosis risk and bone mineral density levels in patients with Parkinson's disease: a meta-analysis. Bone 52:498-505

9. van den Bos F, Speelman AD, van Nimwegen M et al (2013) Bone mineral density and vitamin D status in Parkinson's disease patients. J Neurol 260(3):754-760

10. Walker RW, Chaplin A, Hancock RL et al (2013) Hip fractures in people with idiopathic Parkinson's disease: incidence and outcomes. Mov Dis: Off J Mov Disord Soc 28:334-340

11. Kanis JA, Johnell O, Oden A et al (2008) FRAX and the assessment of fracture probability in men and women from the
UK. Osteoporos Int: J Establ Result Coop Between Eur Found Osteoporos Natl Osteoporos Found USA 19:385-397

12. Hippisley-Cox J, Coupland C (2012) Derivation and validation of updated QFracture algorithm to predict risk of osteoporotic fracture in primary care in the United Kingdom: prospective open cohort study. BMJ 344:e3427. doi:10.1136/bmj.e3427

13. National Institute for Health and Clinical Excellence (2012) Osteoporosis: assessing the risk of fragility fracture. Clinical Guideline 146. www.nice.org.uk/CG146

14. National Institute for Health and Clinical Excellence (2011) Alendronate, etidronate, risedronate, raloxifene and strontium ranelate for the primary prevention of osteoporotic fragility fractures in postmenopausal women (amended). Clinical Guideline 160. http://www.nice.org.uk/TA160

15. Gibb W, Lees A (1989) The significance of the Lewy body in the diagnosis of idiopathic Parkinson's disease. Neuropathol Appl Neurobiol 15:27-44

16. Taylor KS, Cook JA, Counsell CE (2007) Heterogeneity in male to female risk for Parkinson's disease. J Neurol Neurosurg Psychiatry 78:905-906

17. Cheng K-Y, Lin W-C, Chang W-N et al (2013) Factors associated with fall-related fractures in Parkinson's disease. Parkinsonism Relat Disord 20(1):88-92

18. Kanis JA, McCloskey EV, Johansson $\mathrm{H}$ et al (2008) Case finding for the management of osteoporosis with FRAX - assessment and intervention thresholds for the UK. Osteoporos Int: J Establ Result Coop Between Eur Found Osteoporos Natl Osteoporos Found USA 19:1395-1408

19. Dobson R, Leddy SG, Gangadharan S, Giovannoni G (2013) Assessing fracture risk in people with MS: a service development study comparing three fracture risk scoring systems. BMJ Open 3:e002508. doi:10.1136/bmjopen-2012-002508

20. Christianson MS, Shen W (2013) Osteoporosis prevention and management: nonpharmacologic and lifestyle options. Clin Obstet Gynecol 56(4):703-710 